Check for updates

Cite this: Nanoscale Horiz., 2021, 6,751

Received 30th November 2020, Accepted 20th May 2021

DOI: 10.1039/d0nh00670j

rsc.li/nanoscale-horizons

\section{Controlled deposition of nanoparticles with critical Casimir forces $\dagger$}

\author{
Emanuele Marino, (D) ab Oleg A. Vasilyev, (D) ${ }^{c d}$ Bas B. Kluft, ${ }^{b}$ Milo J. B. Stroink, ${ }^{b}$ \\ Svyatoslav Kondrat (D)*cde and Peter Schall*b
}

\begin{abstract}
Nanocrystal assembly represents the key fabrication step to develop next-generation optoelectronic devices with properties defined from the bottom-up. Despite numerous efforts, our limited understanding of nanoscale interactions has so far delayed the establishment of assembly conditions leading to reproducible superstructure morphologies, therefore hampering integration with large-scale, industrial processes. In this work, we demonstrate the deposition of a layer of semiconductor nanocrystals on a flat and unpatterned silicon substrate as mediated by the interplay of critical Casimir attraction and electrostatic repulsion. We show experimentally and rationalize with Monte Carlo and molecular dynamics simulations how this assembly process can be biased towards the formation of 2D layers or 3D islands and how the morphology of the deposited superstructure can be tuned from crystalline to amorphous. Our findings demonstrate the potential of the critical Casimir interaction to direct the growth of future artificial solids based on nanocrystals as the ultimate building blocks.
\end{abstract}

Semiconductor nanocrystals, quantum dots (QDs), reside at the 1-10 $\mathrm{nm}$ length scale separating atoms and colloids and have the potential of becoming the ultimate building blocks of future artificial solids ${ }^{1}$ by promoting material properties fundamentally defined from the bottom-up. ${ }^{2,3}$ The assembly of QDs into crystalline solids, or supercrystals, is of key importance as it represents the main fabrication step of these

\footnotetext{
${ }^{a}$ Department of Chemistry, University of Pennsylvania, Philadelphia, Pennsylvania 19104, USA

${ }^{b}$ van der Waals-Zeeman Institute, University of Amsterdam, Amsterdam, The Netherlands. E-mail: p.schall@uva.nl

${ }^{c}$ Max-Planck-Institut für Intelligente Systeme, Heisenbergstraße 3,

D-70569 Stuttgart, Germany. E-mail: svyatoslav.kondrat@gmail.com, skondrat@ichf.edu.pl

${ }^{d}$ IV. Institut für Theoretische Physik, Universität Stuttgart, Pfaffenwaldring 57, D-70569 Stuttgart, Germany

${ }^{e}$ Institute of Physical Chemistry, Polish Academy of Sciences, Kasprzaka 44/52, 01-224 Warsaw, Poland

$\dagger$ Electronic supplementary information (ESI) available: A detailed description of experimental and modeling procedures. See DOI: 10.1039/d0nh00670j
}

\begin{abstract}
New concepts
Traditional assembly methods for nanoparticles rely on fast solvent drying, resulting in superstructure morphologies that are difficult to reproduce. In this paper, we take advantage of attractive critical Casimir interactions to get a direct grip on the nanoscale assembly and steer the growth of specific nanoparticle morphologies with external temperature control. Such direct control is so far lacking at the nanometer scale. We demonstrate the flexibility of our method by growing crystalline, amorphous, and spinodal superstructures, as well 2D layers and 3D islands. As the superstructures grow on a silicon substrate, the resulting nanocrystal films may be directly processed into a device structure for applications. In addition, our approach allows new insights into the nature of fluctuations and inter-particle interactions by visualization of the nanoscale footprint of the critical Casimir interactions through the spinodal nanoparticle tiling.
\end{abstract}

complex materials. Of the several assembly techniques investigated, ${ }^{4,5}$ controlled solvent evaporation at the air/liquid interface has led to the largest crystalline domain size of $2 \mathrm{D}$ supercrystals of QDs; ${ }^{6}$ this approach is versatile and compatible with $3 \mathrm{D}$ assembly, ${ }^{7}$ although the transfer of the crystalline QD layer to a solid substrate can lead to film cracking, ${ }^{8}$ hampering scalability. There is, therefore, the need to improve the integration of QD supercrystals onto planar substrates. An ideal solution would be controlling both QD-QD and QD-substrate interactions to drive the growth of QD supercrystals directly onto a desired substrate.

Critical Casimir interactions have attracted much attention recently as a means to steer the assembly of micro-scaled colloids and nanoparticles dissolved in a binary solvent close to its critical point. ${ }^{9-15}$ The confinement of solvent fluctuations between particles causes a net attractive force driving the assembly process. ${ }^{15-17}$ Changing the distance from the critical point regulates the size and composition of these fluctuations, thereby controlling the magnitude and the range of the Casimir interaction. Consequently, the temperature of the near-critical solvent represents a physical knob to finely tune the particle interactions, favoring interaction-dependent superstructure morphologies. ${ }^{14}$ Similarly, the confinement of solvent fluctuations 
between particles and a substrate also leads to particle-substrate interactions that can ultimately result in controllable adsorption of particles on a substrate. ${ }^{18-22}$ In the case of nanoparticles, this can allow for controlled nanocrystal deposition analogous to atomic deposition techniques such as chemical vapor deposition, with the advantage that the nanocrystal interactions can be directly controlled by temperature. While for typically-used micrometer-sized colloidal particles the temperature needs to be inconveniently close to the critical temperature, $T_{\mathrm{c}}$, by several tens of millikelvin to reach interaction ranges comparable to the colloid size, for nanoparticles the temperature range widens up to ten Kelvin below $T_{\mathrm{c}}$, making this technique more easily applicable. ${ }^{12}$

Herein, we demonstrate the controlled deposition of QDs onto a silicon substrate driven by critical Casimir forces. By tuning the interplay between the critical Casimir attraction and electrostatic repulsion, we direct the growth of patches of a crystalline monolayer of QDs on a substrate. We show how the morphology of deposited QD superstructures can be varied from crystalline to amorphous and from layer-by-layer to island growth by navigating through the multidimensional parameter space of Debye screening length, substrate wetting properties and solvent correlation length. To gain physical insights into the assembly process, we perform 3D Monte Carlo (MC) simulations of the Ising model, which mimics a binary liquid mixture close to criticality. To connect simulations and experiments, we measure the size of the solvent fluctuations in situ and compute the critical Casimir interaction potentials for the experimentally relevant configurations. Our results open up new directions for the assembly and deposition control of QDS at nanometer length scales, demonstrating conveniently tunable nanoparticle interactions.

We prepare cadmium selenide (CdSe) QDs with average diameter $d_{\mathrm{QD}}=5.5 \mathrm{~nm}$ and passivated by hydrophilic 2 , 3-dimercapto-1-propanesulfonate (DMPS) ligands, and disperse them in a binary mixture of lutidine and water with lutidine mass fraction $c_{\mathrm{L}}=0.3$, just above the critical concentration $c_{\mathrm{c}}=0.286$. Since the presence of QDs and free ligands in solution influences the binary liquid phase diagram slightly, we experimentally measure the phase diagram of the nanocrystal-containing binary mixture. To induce attractive critical Casimir interactions at a substrate, we heat the substrate to the desired temperature below $T_{\mathrm{c}}, T=T_{\mathrm{c}}-\Delta T$. The heated substrate is submerged into the solution from above to avoid particle clusters assembling in the bulk of the solvent from sedimenting onto it and to minimize convection, see Fig. 1a. The solution is in thermal contact with a bath at room a

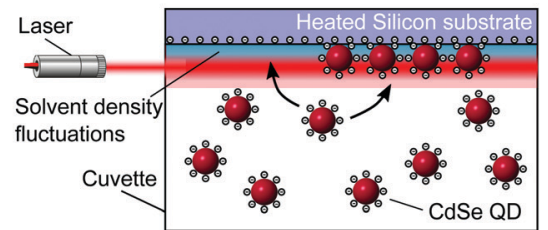

C

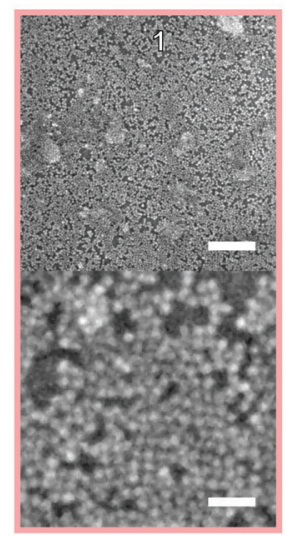

b
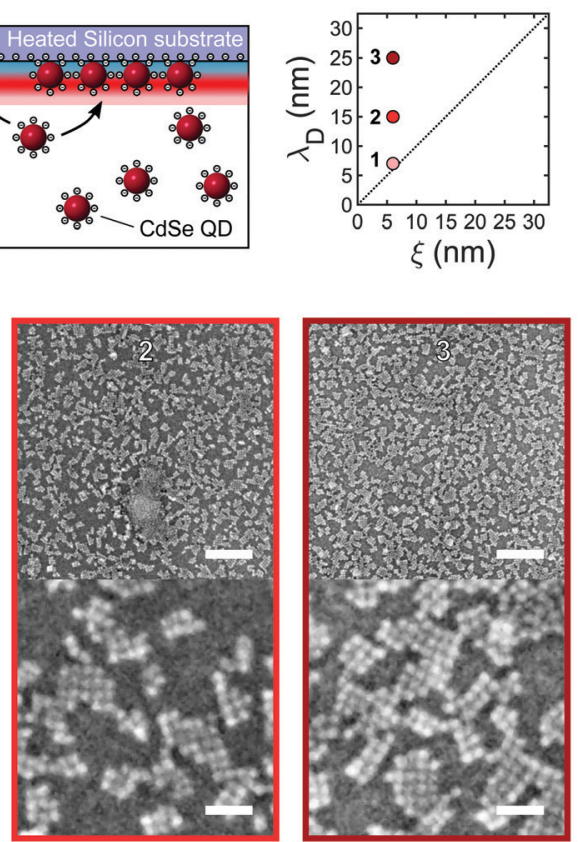

d

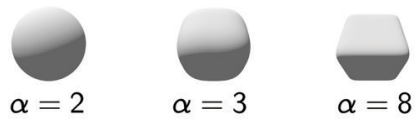

e

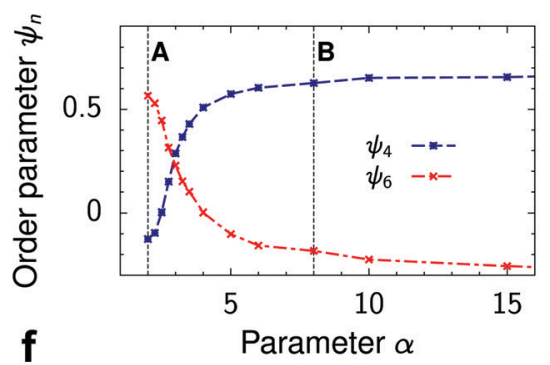

f
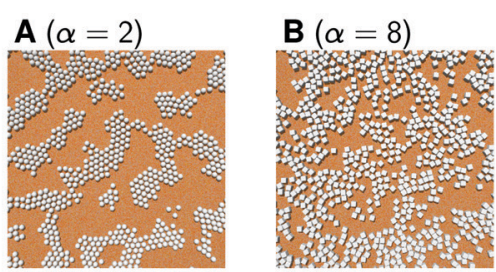

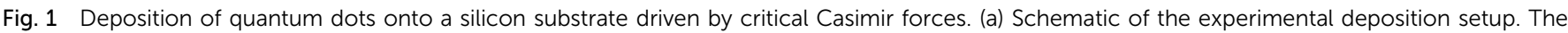

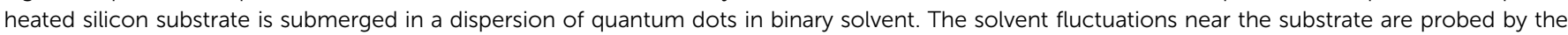

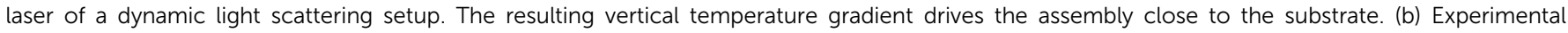

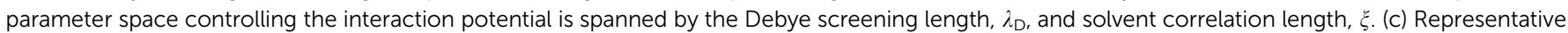

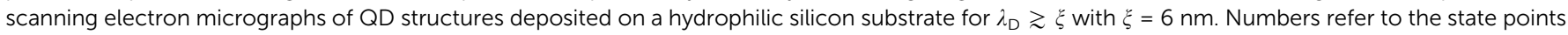

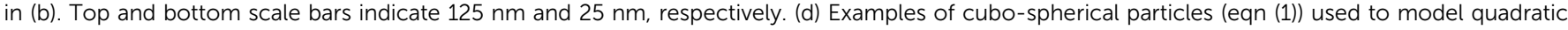

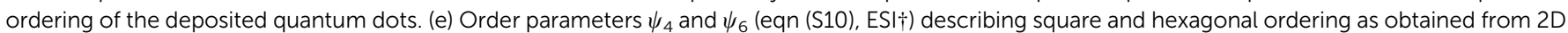

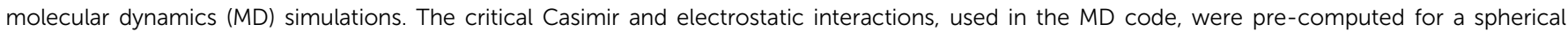

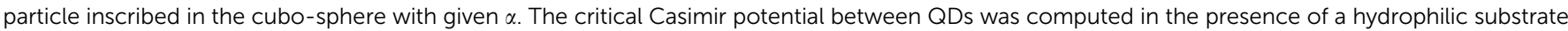

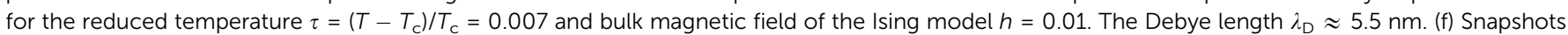
from the MD simulations demonstrating (A) hexagonal and (B) quadratic ordering, corresponding to $\alpha=2$ and $\alpha=8$. See also videos (ESI $\dagger$ ). 
temperature, establishing a temperature gradient away from the heated substrate (see Fig. S2 (ESI $\dagger$ ) for the calculated temperature distribution). We measure the temperaturedependent solvent correlation length $\xi$ directly using dynamic light scattering by aligning the laser beam of a light scattering setup (ALV-6000/60X0) close to the substrate so that scattering results from the solvent fluctuations near the surface. This allows us to directly measure the crucial parameter determining the attraction, $\xi .{ }^{13}$ Due to the slightly off-critical composition, $\xi$ does not diverge upon approaching the critical temperature, but increases strongly to correlation lengths substantially larger than the molecular dimension. ${ }^{13}$

While the magnitude and range of the critical Casimir attraction depend on the size of the solvent composition fluctuations, $\xi(\Delta T),{ }^{9,15,16,18}$ the magnitude and extent of the electrostatic repulsion depend on the Debye screening length $\lambda_{\mathrm{D}}$, set by the concentration of free ions in solution. ${ }^{23}$ Since the dielectric constant of the QDs and silicon substrate $(\varepsilon \approx 5-6.25$, see ref. 24 and 25) is comparable to the dielectric constant of the near-critical water-lutidine $(\varepsilon \approx 7$, see ref. 26$)$, the van der Waals interactions are weak and hence the behaviour of the QDs at a substrate is mostly determined by the interplay of the electrostatic and critical Casimir interactions. The corresponding experimental parameter space of $\xi$ and $\lambda_{\mathrm{D}}$ is shown in Fig. $1 b$.

With increasing exposure time in the QD suspension, the silicon substrate exhibits an increasing amount of deposited material (Fig. S3, ESI $\dagger$ ), suggesting the continuous deposition of a QD film. The assembly also appears general yet sensitive to the choice of substrate, as exemplified by depositions on a gold substrate (Fig. S4, ESI $\dagger$ ). We focus on short deposition times of 1 hour on silicon and investigate the structure and morphology of the deposits. At sufficiently low attraction, $\lambda_{\mathrm{D}}>\xi$, the substrate after drying contains ordered crystalline patches of QDs (Fig. 1c). Drawing an analogy with atomic deposition processes, ${ }^{27}$ the deposition of planar structures suggests that the attraction between a QD and the substrate is larger than that between the QDs; furthermore, the overall low attraction magnitude allows the particles to form an equilibrium crystalline phase. Surprisingly, the crystalline patches exhibit square packing featuring four nearest neighbors, instead of six neighbors characterizing the hexagonal packing. The square packing may arise due to QD facets, relating to their zinc blende atomic lattice, emphasized by passivation with short DMPS ligands.

To assess whether such cubic-like shapes could lead to the formation of square lattices, we model the deposited particles as rounded cubes described by

$$
\left(\frac{x}{R}\right)^{\alpha}+\left(\frac{y}{R}\right)^{\alpha}+\left(\frac{z}{R}\right)^{\alpha}=1
$$

where $\alpha$ is a parameter defining the shape and $R=d_{\mathrm{QD}} / 2$ is the radius of a particle. For $\alpha=2$, eqn (1) describes a spherical particle, which continuously transforms into a cube as $\alpha$ increases (Fig. 1d). Taking into account the short-range, critical Casimir and electrostatic interactions between such rounded cubes (Section S4, ESI $\dagger$ ), we minimize the energy of QDS arranged on two lattices and find that the square lattice is favourable for $\alpha>\alpha_{0} \approx 2.71$ (Fig. S7, ESI $\dagger$ ). To elucidate the structural transition in more detail, we compute bond order parameters $\psi_{4}$ and $\psi_{6}$ that take up values close to 1 for square and hexagonal ordering, respectively (Section S4C, ESI $\dagger$ ). We perform 2D molecular dynamics (MD) simulations for particles with various values of $\alpha$ (Section S4B, ESI $\dagger$ ), and show the resulting order parameters and structures as a function of $\alpha$ in Fig. 1e and f. The quadratic order parameter $\psi_{4}$ increases abruptly as $\alpha$ increases from $\alpha=2$ and dominates for $\alpha \gtrsim 2.8$, suggesting that the shape of a QD is likely responsible for the quadratic ordering seen in the experiments. Our simulations also indicate that a transformation from the hexagonal to square lattice can be induced by varying the Debye screening
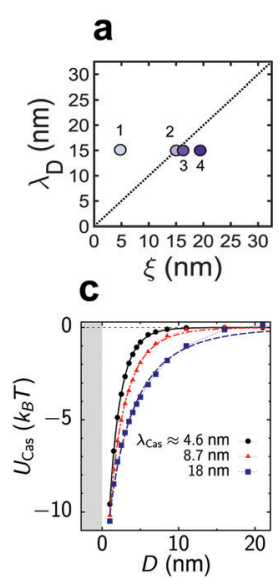

b

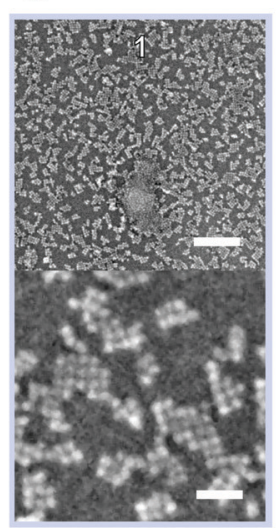

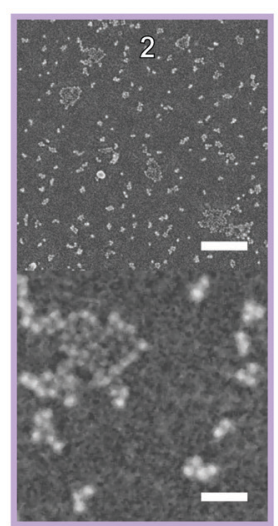

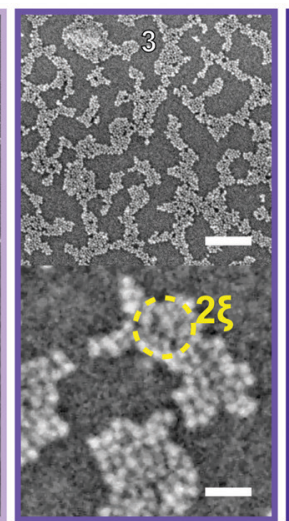

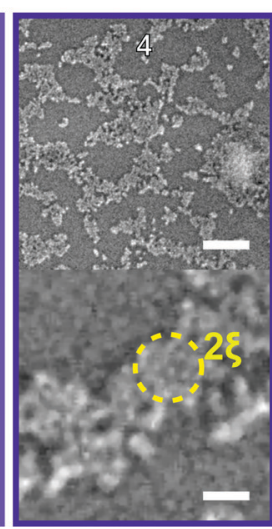

Fig. 2 QD deposition on a hydrophilic silicon substrate for fixed Debye screening length. (a) Schematic of the available parameter space and the values of the measured correlation length, $\xi$, and Debye screening length, $\lambda_{\mathrm{D}}$, used in (b). (b) Representative scanning electron micrographs for the states indicated in (a). Top and bottom scale bars indicate $125 \mathrm{~nm}$ and $25 \mathrm{~nm}$, respectively. Dashed circles indicate two times the correlation length for orientation. (c) Attractive critical Casimir potential between a QD and the substrate obtained by Monte Carlo (MC) simulations of the Ising model. $D$ is the surface-to-surface distance and $\lambda_{\text {cas }} \approx \xi$ is the decay length of the Casimir potential obtained by fitting it to the exponentially decaying function of $D$ (Section S3A, ESI†). The grey area shows the region of steric exclusion. 
length (Fig. S8, ESI $\dagger$ ). It will be interesting to investigate such transformations in more detail in future work.

When decreasing the Debye screening length to reduce the repulsive component to $\lambda_{\mathrm{D}} \sim \xi$, the crystalline structure dissolves in favor of amorphous deposits (Fig. 1c). A similar loss of crystallinity is observed when we increase the attractive length scale while keeping the repulsion fixed: The initially crystalline deposits become increasingly amorphous as shown in Fig. 2. In both cases, we increase the attractive character of the effective particle-particle and particle-substrate interactions that result from the superposition of the screened electrostatic repulsion and the critical Casimir attraction. We conclude that the structure can no longer rearrange to reach the lowest-energy configuration. We can thus tune the structure of the deposit by either adjusting the salt concentration or temperature to modulate the QD-substrate interaction. Furthermore, controlling the wetting properties of the substrate offers an orthogonal handle over the assembly behaviour, as we will show below. We note that our 2D multiscale simulations ${ }^{28}$ do not show this loss of crystalline order with decreasing $\lambda_{\mathrm{D}}$ but rather the opposite trend; we therefore associate it with ligand or surface friction effects that slow down the particle dynamics at increasing attraction.

The increasing solvent correlation length also leads to a key morphological feature: As the size of the solvent fluctuations increases, the QD aggregates coarsen and assume a welldefined morphology, reminiscent of spinodal patterns. Such spinodal morphologies are characteristic of two-component mixtures subjected to rapid demixing. ${ }^{29}$ Indeed, the size of the observed structures is of the order of $2 \xi$ (dashed circles in Fig. 2b) and increases with increasing $\xi$ (from left to right in
Fig. 2b). Similar spinodal structures are also obtained in our multi-scale molecular dynamics simulations of deposited nanoparticles. ${ }^{28}$

As opposed to micrometer-sized colloidal particles, the solvent fluctuations can reach sizes comparable to or larger than the QD diameter even at temperatures far below $T_{\mathrm{c}}$. By increasing the solvent correlation length from $\xi=5 \mathrm{~nm}<d_{\mathrm{QD}}$ to $\xi \geq 15 \mathrm{~nm}>d_{\mathrm{QD}}$, we invert the ratio of $\xi$ to the particle diameter $\mathrm{d}_{\mathrm{QD}}$. The case $\xi / d_{\mathrm{QD}}<1$ is typical for micronsized colloids and has been extensively studied in the literature. ${ }^{10,11,13,15,18,30,31}$ For $\xi / d_{\mathrm{QD}}>1$, many-body interactions between the QDs likely play a dominant role. ${ }^{32,33}$ Fig. $2 b$ suggest that, in this case, the QDs adsorbed onto the substrate follow the specific patterns of the solvent fluctuations. Thus, besides nanoparticle deposition, our experimental approach can be used to deepen the fundamental understanding of colloidal interactions by using nanoparticles as spatial probes.

The increasing correlation length also leads to a nontrivial change of the amount of deposited material, namely, with increasing $\xi$ the surface coverage changes in a nonmonotonic way (Fig. 2b). The critical Casimir potential $\mathrm{U}_{\text {Cas }}$ shown in Fig. 2c cannot explain this surprising behaviour because its magnitude increases with increasing decay length $\lambda_{\text {Cas }} \approx \xi$. The reduced surface coverage in Fig. 2 suggests that the increasing correlation length enhances the surface charge density due to the increased water content at the substrate, hence amplifying the QD-substrate electrostatic repulsion. For the larger correlation lengths ( $\xi \gtrsim 18 \mathrm{~nm}$ in Fig. $2 \mathrm{~b}$ ), the critical Casimir forces eventually start to dominate the interactions, leading to an increase in surface coverage. While microscopic theories and complementary experiments are needed to assess

\section{a}
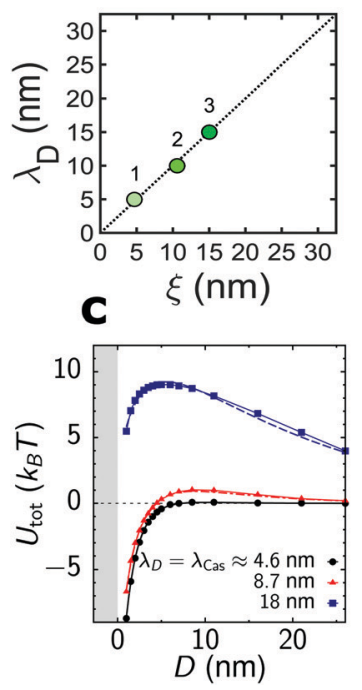

b

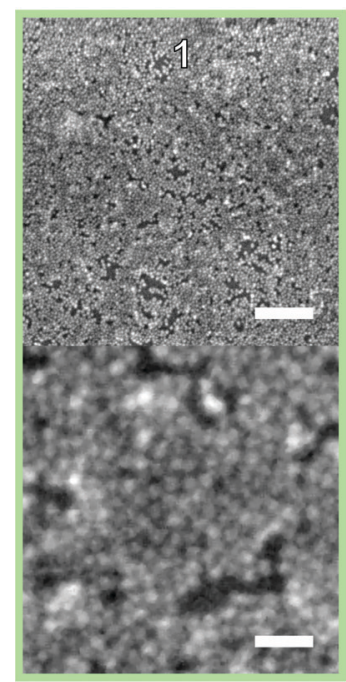

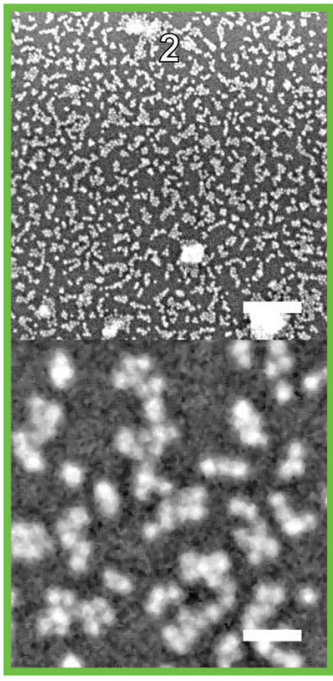

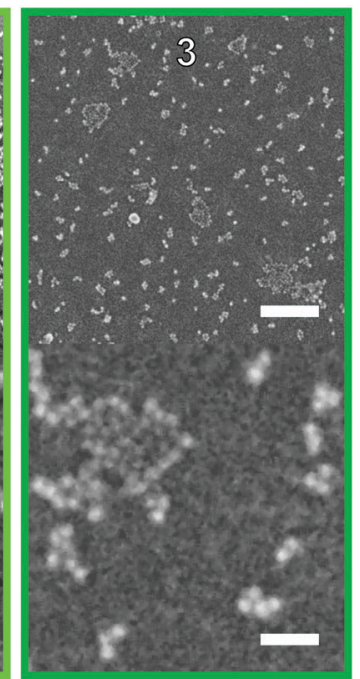

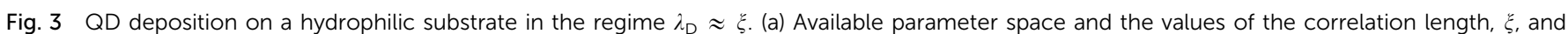

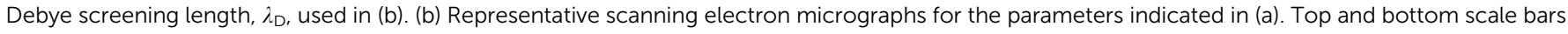

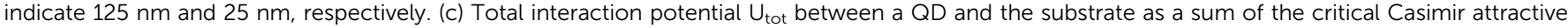

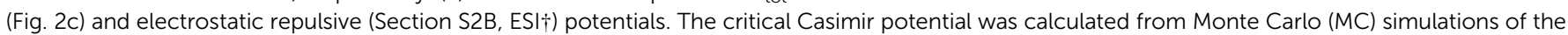
Ising model and its decay length $\lambda_{\text {Cas }} \approx \xi$ was obtained by fitting the MC data to an exponentially decaying function of separation. 
this hypothesis, we note that such unusual effects have been previously reported for other types of surfaces. For equallycharged surfaces with unlike preferences for the fluid component (e.g., hydrophobic substrates and hydrophilic particles), there is a temperature-induced crossover between attractive and repulsive forces ${ }^{34}$ that arises due to the subtle interplay between the electrostatic and critical Casimir forces. ${ }^{35-37}$ Similarly, oppositely-charged hydrophobic surfaces can repel each other, even though separately both the critical Casimir and electrostatic forces are attractive. ${ }^{38}$

When we increase the range of the attraction and repulsion simultaneously, keeping their ratio constant, we observe a substantial decrease in the surface coverage (Fig. 3a and b). The strength of the electrostatic repulsion increases quadratically with the Debye screening length (Section S2B, eqn (S6), ESI $\dagger$ ), while the magnitude of the critical Casimir attraction varies only weakly with the correlation length (Fig. 2c). This leads to an energy barrier for QDs approaching the substrate when $\lambda_{\mathrm{D}}=\xi$ increases (Fig. 3c), which reduces the surface coverage. A similar effect has been reported for the assembly of micro-scale colloids, which yields the aggregation line well below the $\lambda_{\mathrm{D}}=\xi$ line. $^{39}$

We finally bias the assembly process to selectively drive QD island growth. Atomic deposition methods describe island growth as characterized by interparticle interactions stronger than particle-substrate interactions. We achieve these conditions by engineering the surface affinity of the silicon substrate from hydrophilic to hydrophobic by treating the substrate with 3-mercaptopropyl trimethoxysilane, ${ }^{40}$ resulting in a contact angle of $74^{\circ}$ with water, see Fig. S1 (ESI $\dagger$ ). The resulting deposits indeed exhibit island morphology, as shown in Fig. 4b.

We thus tuned the deposition of 2D QD layers towards 3D island growth, with island sizes between 50 and $500 \mathrm{~nm}$. Yet, it is surprising that we observe any superstructure growth at all as the critical Casimir force becomes repulsive in the presence of asymmetric boundary conditions, namely hydrophobic substrate and hydrophilic particles. ${ }^{18}$ Such islands may grow on locations of the substrate where the hydrophobic surface coverage is defective, therefore exposing hydrophilic patches (Fig. 4c). To understand how the presence of such patches

\section{a}
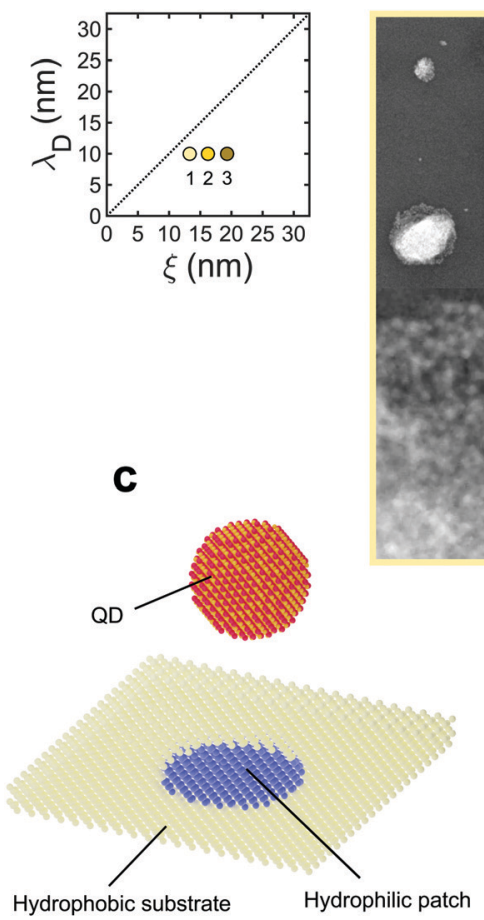

b
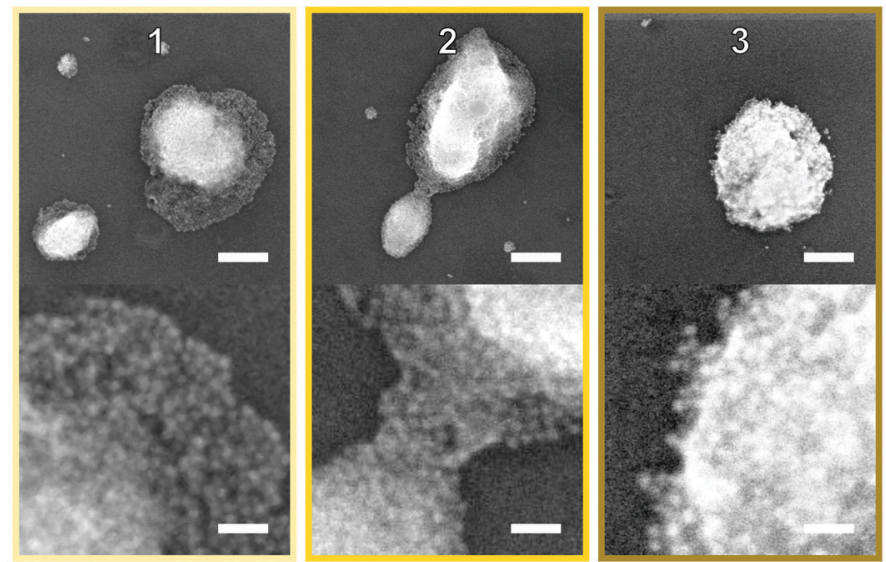

e
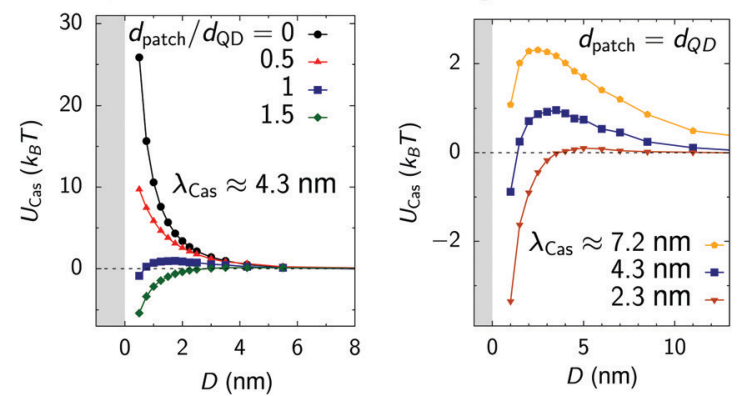

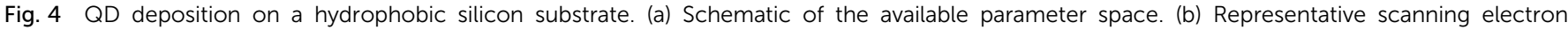

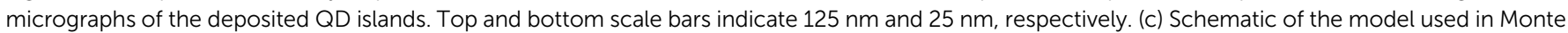

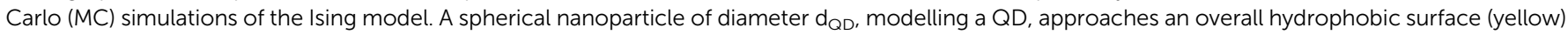

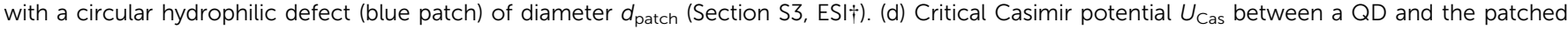

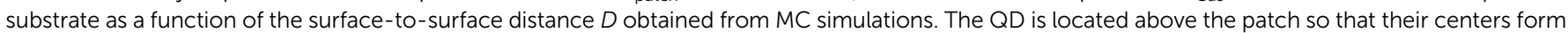

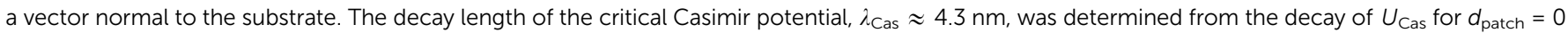

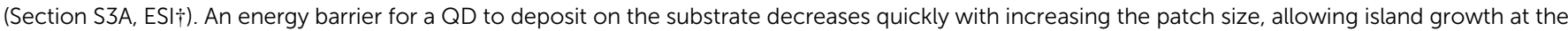

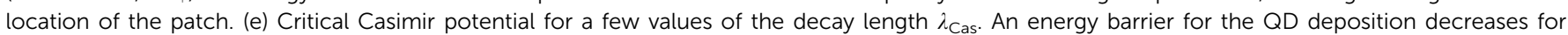
decreasing $\lambda_{\text {Cas, }}$ enabling more deposition islands to form on the substrate. 
affects the critical Casimir interactions between a QD and the substrate, we performed MC simulations for various patch sizes $d_{\text {patch }}$ Fig. $4 \mathrm{~d}$ shows that the energy barrier for QD deposition decreases rapidly with increasing $d_{\text {patch }}$ and practically vanishes when the patch size becomes comparable to the QD size. Hence, deposition of a single QD may result in a cascade reaction that will quickly lead to the formation of an island. Our simulations also reveal that the energy barrier decreases for decreasing decay length of the critical Casimir interactions (Fig. 4e). The decreasing range of interactions weakens the effect of the hydrophobic substrate on a QD that is located exactly above the hydrophilic patch, thus weakening the repulsion. This explains why more islands form for smaller correlation lengths. Such island formation due to hydrophilic patches demonstrates the potential of our method for fabricating complex nanoparticle structures via substrate patterning.

In conclusion, our experiments and simulations demonstrate that via direct particle interaction control at the nanoscale, the critical Casimir effect allows the deposition of QDs on a substrate in an administering manner. Application of this effect to nanometer-size particles offers convenient tuning of their interactions in a temperature window of more than $10 \mathrm{~K}$, a range much larger than in previous studies on micron-scale colloids. ${ }^{10,11,15,18,30}$ By varying the Casimir attraction and the electrostatic repulsion, we obtain various morphologies ranging from crystalline to spinodal. We expect this effect to be general to any nanoparticle that can be charge stabilised in the binary mixture; the magnitude of the repulsive and attractive components will then be set by the surface charges of particles and substrate, and their lutidine/water affinities, respectively. Furthermore, by changing the substrate wetting properties, we direct the growth from 2D layers to 3D islands (Fig. 1,4, respectively). Our experiments also uncover physically unexpected results such as the formation of quadratic, rather than hexagonal, lattices (Fig. 1) and a surprising non-monotonic behavior of surface coverage with the solvent correlation length (Fig. 2).

We envision that our method may lead to faster and cheaper fabrication techniques for optoelectronic devices based on nanocrystal self-assembly. Combined with chemical substrate patterning, the critical Casimir-driven nanoparticle deposition can produce desired structures, challenging to fabricate otherwise. These well-defined morphologies can find a broad range of applications, such as light-emitting diode displays with nanocrystal-sized pixels. Our approach may also serve as the testing ground to deepen our knowledge of fluctuation phenomena and critical Casimir forces at the nanoscale to experimentally assess the effects predicted by theory. ${ }^{15,28,33,41-45}$

\section{Methods}

\section{Experiments}

We have synthesized CdSe QDs by following a procedure reported in the literature. ${ }^{46}$ The hydrophobic, oleate-capped, QDs were ligand-exchanged for hydrophilic DMPS ligands by using an equal volume hexane/N-methyl formamide two-phase system featuring an excess of 1000 ligands per QD. After washing with acetonitrile, the ligand-exchanged QDs were redispersed in the binary mixture of 2,6-lutidine in water.

The QD dispersion was then transferred to a custom-made glass cuvette for light-scattering which was placed in the sample holder for a standard dynamic light scattering system (ALV-6000/60X0 Multiple Tau Digital Real Correlator), a decaline bath at room temperature. A silicon substrate, made either hydrophilic through plasma-cleaning or hydrophobic through silanization (see ref. 40, method 2), was placed in contact with the QD dispersion by immersion. The silicon substrate was in thermal contact with a steel block. The steel block was then heated to the desired temperature by means of an embedded thermistor.

The solvent correlation length $\xi$ was directly measured by using dynamic light scattering after aligning the He-Ne laser beam (wavelength $632.8 \mathrm{~nm}$, power $22 \mathrm{~mW}$ ) parallel and close to the submerged substrate. Specifically, we carefully lowered the cuvette containing the nanocrystal dispersion in the binary mixture in contact with the heating block until the laser beam just cleared of the substrate. The single exponential decay time $\tau$ of the scattered intensity correlation curve is related to the hydrodynamic diameter $d_{\mathrm{H}}$ of the scattering objects undergoing diffusion by $\xi:=d_{\mathrm{H}}=\left(k_{\mathrm{B}} T / 3 \pi \eta\right) q^{2} \tau$, where $k_{\mathrm{B}}$ Boltzmann's constant, $T$ the temperature, $\eta$ is the solvent viscosity, and $q$ is the scattering wavevector. The Debye screening length $\lambda_{\mathrm{D}}$ of the binary mixture was controlled by adjusting the concentration of $\mathrm{NaCl}$ according to $\lambda_{\mathrm{D}}=\sqrt{\left(\varepsilon_{\mathrm{r}} \varepsilon_{0} k_{\mathrm{B}} T\right) /\left(2 \times 10^{3} N_{\mathrm{A}} e^{2} I\right)}$, where $\varepsilon_{\mathrm{r}}$ is the volume-averaged dielectric constant of the solvent mixture, $\varepsilon_{0}$ the permittivity of free space, $N_{\mathrm{A}}$ Avogadro's number, $e$ the elementary charge, and I the molar concentration of $\mathrm{NaCl}$. The depositions shown in the main text lasted one hour. A more extensive description of these procedures can be found in Section S1 (ESI $\dagger$ ).

\section{Monte Carlo simulations}

We have used 3D Monte Carlo (MC) simulations of the Ising model, which mimics a near-critical binary solvent, to compute critical Casimir interaction potentials between a QD and a substrate and between two QDs at a substrate. QDs were modeled as spherical particles on a lattice using the method developed by Vasilyev ${ }^{47}$ (Section S3A, ESI $\dagger$ ). To mimic the experimental conditions, where an off-critical concentration was kept fixed, we varied the values of the reduced temperature and external magnetic field in the Ising model such as to have a constant non-zero magnetization in the bulk (Section S3B, $\mathrm{ESI} \dagger)$.

\section{Molecular dynamics simulations}

We performed 2D molecular dynamics simulations of deposited cubo-spherical particles with the shape defined by eqn (1). The shape was taken into account only on the level of shortrange repulsive interactions (Section S4, ESI $\dagger$ ). The electrostatic and critical Casimir forces were calculated for a sphere 
inscribed in the corresponding cubo-spherical particle. For electrostatic interactions, we used the DLVO theory (Section $\mathrm{S} 2 \mathrm{~A}, \mathrm{ESI} \dagger)$ and the critical Casimir potentials were computed by Monte Carlo simulations of the Ising model, as described above.

\section{Conflicts of interest}

There are no conflicts to declare.

\section{Acknowledgements}

This work is part of the research programme "Nanoarchitectures: Smart Assembly, Quantum Electronics and Soft Mechanics" with project number 680.47.615, which is financed by the Dutch Research Council (NWO). P. S. acknowledges financial support from NWO through personal Vici grant. The authors acknowledge Dr Yingying Tang for carrying out the contact angle measurements.

\section{References}

1 T. Hanrath, Colloidal nanocrystal quantum dot assemblies as artificial solids, J. Vac. Sci. Technol., A, 2012, 30, 030802.

2 M. Cargnello, A. C. Johnston-Peck, B. T. Diroll, E. Wong, B. Datta, D. Damodhar, V. V. T. Doan-Nguyen, A. A. Herzing, C. R. Kagan and C. B. Murray, Substitutional doping in nanocrystal superlattices, Nature, 2015, 524, 450.

3 E. Marino, A. Sciortino, A. Berkhout, K. E. MacArthur, M. Heggen, T. Gregorkiewicz, T. E. Kodger, A. Capretti, C. B. Murray, A. F. Koenderink, F. Messina and P. Schall, Simultaneous Photonic and Excitonic Coupling in Spherical Quantum Dot Supercrystals, ACS Nano, 2020, 14(10), 13806-13815.

4 K. J. M. Bishop, C. E. Wilmer, S. Soh and B. A. Grzybowski, Nanoscale Forces and Their Uses in Self-Assembly, Small, 2009, 5, 1600-1630.

5 M. A. Boles, M. Engel and D. V. Talapin, Self-Assembly of Colloidal Nanocrystals: From Intricate Structures to Functional Materials, Chem. Rev., 2016, 116, 11220-11289.

6 A. Dong, J. Chen, P. M. Vora, J. M. Kikkawa and C. B. Murray, Binary nanocrystal superlattice membranes self-assembled at the liquid-air interface, Nature, 2010, 466, 474-477.

7 E. Marino, T. E. Kodger, G. H. Wegdam and P. Schall, Revealing Driving Forces in Quantum Dot Supercrystal Assembly, Adv. Mater., 2018, 30, 1803433.

8 B. T. Diroll, X. Ma, Y. Wu and C. B. Murray, Anisotropic Cracking of Nanocrystal Superlattices, Nano Lett., 2017, 17, 6501-6506.

9 D. Bonn, J. Otwinowski, S. Sacanna, H. Guo, G. Wegdam and P. Schall, Direct observation of colloidal aggregation by critical Casimir forces, Phys. Rev. Lett., 2009, 103, 156101.

10 V. D. Nguyen, S. Faber, Z. Hu, G. H. Wegdam and P. Schall, Controlling colloidal phase transitions with critical Casimir forces, Nat. Commun., 2013, 4, 1584.
11 P. B. Shelke, V. Nguyen, A. Limaye and P. Schall, Controlling colloidal morphologies by critical Casimir forces, Adv. Mater., 2013, 25, 1499-1503.

12 E. Marino, T. E. Kodger, J. B. ten Hove, A. H. Velders and P. Schall, Assembling quantum dots via critical Casimir forces, Sol. Energy Mater. Sol. Cells, 2016, 158, 154-159.

13 S. G. Stuij, M. Labbé-Laurent, T. E. Kodger, A. Maciołek and P. Schall, Critical Casimir interactions between colloids around the critical point of binary solvents, Soft Matter, 2017, 13, 5233-5249.

14 E. Marino, D. M. Balazs, R. W. Crisp, D. Hermida-Merino, M. A. Loi, T. E. Kodger and P. Schall, Controlling Superstructure-Property Relationships via Critical Casimir Assembly of Quantum Dots, J. Phys. Chem. C, 2019, 123, 13451-13457.

15 A. Maciołek and S. Dietrich, Collective behavior of colloids due to critical Casimir interactions, Rev. Mod. Phys., 2018, 90, 045001.

16 M. E. Fisher and P.-G. de Gennes, Phénomènes aux parois dans un mélange binaire critique, CR Acad. Sci. Ser. B, 1978, 287, 207-209.

17 A. Gambassi, The Casimir effect: From quantum to critical fluctuations, J. Phys.: Conf. Ser., 2009, 161, 012037.

18 C. Hertlein, L. Helden, A. Gambassi, S. Dietrich and C. Bechinger, Direct measurement of critical Casimir forces, Nature, 2008, 451, 172-175.

19 M. Tröndle, S. Kondrat, A. Gambassi, L. Harnau and S. Dietrich, Normal and lateral critical Casimir forces between colloids and patterned substrates, EPL, 2009, 88, 40004.

20 M. Tröndle, S. Kondrat, A. Gambassi, L. Harnau and S. Dietrich, Critical Casimir effect for colloids close to chemically patterned substrates, J. Chem. Phys., 2010, 133, 074702 .

21 M. Tröndle, O. Zvyagolskaya, A. Gambassi, D. Vogt, L. Harnau, C. Bechinger and S. Dietrich, Trapping colloids near chemical stripes via critical Casimir forces, Mol. Phys., 2011, 109, 1169-1185.

22 A. Gambassi and S. Dietrich, Critical Casimir forces steered by patterned substrates, Soft Matter, 2011, 7, 1247-1253.

$23 \mathrm{~J} . \mathrm{N}$. Israelachvili, Intermolecular and surface forces, Academic press, 2011.

24 U. Pal, D. Samanta, S. Ghorai and A. K. Chaudhuri, Optical constants of vacuum-evaporated polycrystalline cadmium selenide thin films, J. Appl. Phys., 1993, 74, 6368-6374.

25 T. Wakaoka, K. Hirai, K. Murayama, Y. Takano, H. Takagi, S. Furukawa and S. Kitagawa, Confined synthesis of CdSe quantum dots in the pores of metal-organic frameworks, J. Mater. Chem. C, 2014, 2, 7173-7175.

26 F. Pousaneh, O. Edholm and A. Maciołek, Molecular dynamics simulation of a binary mixture near the lower critical point, J. Chem. Phys., 2016, 145, 014501.

27 I. V. Markov, Crystal Growth for Beginners, World Scientific, 2018, p. 632.

28 O. A. Vasilyev, E. Marino, B. B. Kluft, P. Schall and S. Kondrat, Debye vs. Casimir: controlling the structure of 
charged nanoparticles deposited on a substrate, Nanoscale, 2021, 13, 6475-6488.

29 E. M. Herzig, K. A. White, A. B. Schofield, W. C. K. Poon and P. S. Clegg, Bicontinuous emulsions stabilized solely by colloidal particles, Nat. Mater., 2007, 6, 966.

30 D. Beysens and D. Estève, Adsorption Phenomena at the Surface of Silica Spheres in a Binary Liquid Mixture, Phys. Rev. Lett., 1985, 54, 2123-2126.

31 A. Magazzù, A. Callegari, J. P. Staforelli, A. Gambassi, S. Dietrich and G. Volpe, Controlling the dynamics of colloidal particles by critical Casimir forces, Soft Matter, 2019, 15, 2152-2162.

32 S. Paladugu, A. Callegari, Y. Tuna, L. Barth, S. Dietrich, A. Gambassi and G. Volpe, Nonadditivity of critical Casimir forces, Nat. Commun., 2016, 7, 11403.

33 O. A. Vasilyev, S. Dietrich and S. Kondrat, Nonadditive interactions and phase transitions in strongly confined colloidal systems, Soft Matter, 2018, 14, 586-596.

34 U. Nellen, J. Dietrich, L. Helden, S. Chodankar, K. Nygård, J. F. van der Veen and C. Bechinger, Salt-induced changes of colloidal interactions in critical mixtures, Soft Matter, 2011, 7, 5360.

35 F. Pousaneh and A. Ciach, The origin of the attraction between like charged hydrophobic and hydrophilic walls confining a near-critical binary aqueous mixture with ions, J. Phys.: Condens. Matter, 2011, 23, 412101.

36 M. Bier, A. Gambassi, M. Oettel and S. Dietrich, Electrostatic interactions in critical solvents, EPL, 2011, 95, 60001.

37 F. Pousaneh, A. Ciach and A. Maciołek, Effect of ions on confined near-critical binary aqueous mixture, Soft Matter, 2012, 8, 7567.
38 F. Pousaneh, A. Ciach and A. Maciołek, How ions in solution can change the sign of the critical Casimir potential, Soft Matter, 2014, 10, 470-483.

39 A. Gambassi and S. Dietrich, Colloidal Aggregation and Critical Casimir Forces, Phys. Rev. Lett., 2010, 105, 059601.

40 J. Cras, C. Rowe-Taitt, D. Nivens and F. Ligler, Comparison of chemical cleaning methods of glass in preparation for silanization, Biosens. Bioelectron., 1999, 14, 683-688.

41 T. F. Mohry, S. Kondrat, A. Maciołek and S. Dietrich, Critical Casimir interactions around the consolute point of a binary solvent, Soft Matter, 2014, 10, 5510-5522.

42 S. Kondrat, O. A. Vasilyev and S. Dietrich, Probing interface localization-delocalization transitions by colloids, J. Phys.: Condens. Matter, 2018, 30, 414002.

43 K. Marolt, M. Zimmermann and R. Roth, Microphase separation in a two-dimensional colloidal system with competing attractive critical Casimir and repulsive magnetic dipole interactions, Phys. Rev. E, 2019, 100, 052602.

44 O. A. Vasilyev, M. Labbé-Laurent, S. Dietrich and S. Kondrat, Bridging transitions and capillary forces for colloids in a slit, J. Chem. Phys., 2020, 153, 014901.

45 N. F. Bafi, P. Nowakowski and S. Dietrich, Effective pair interaction of patchy particles in critical fluids, J. Chem. Phys., 2020, 152, 114902.

46 B. D. Chernomordik, A. R. Marshall, G. F. Pach, J. M. Luther and M. C. Beard, Quantum Dot Solar Cell Fabrication Protocols, Chem. Mater., 2017, 29, 189-198.

47 O. A. Vasilyev, Critical Casimir interactions between spherical particles in the presence of bulk ordering fields, Phys. Rev. E, 2014, 90, 012138. 